\title{
Robotic single-incision anterior resection for sigmoid colon cancer: access port creation and operative technique
}

\author{
Meng Shi Lim • George Melich • Byung Soh Min
}

Received: 3 November 2011 / Accepted: 30 July 2012/Published online: 10 October 2012

(C) Springer Science+Business Media, LLC 2012

\begin{abstract}
Background Potential morbidities related to multiport laparoscopic surgeries have led to the current excitement about single-incision laparoscopic techniques. However, multiport laparoscopy is technically demanding and ergonomically challenging. We present our technique of using the Alexis wound retractor and a surgical glove to fashion an access port and the da Vinci surgical robot to perform single-incision anterior resection.

Methods Through a small transumbilical incision, an Alexis wound retractor and a surgical glove are fashioned as an access port. Appropriate trocars are then inserted through the cut fingertips of the glove and secured. A threearm da Vinci robot with a $30^{\circ}$ up-scope was used.

Results Twenty-two patients (12 males, 10 females) with a mean age of 58.5 years (range $=35-70$ ) underwent robotic single-incision anterior resection for sigmoid colon cancer with this technique. There was no conversion to
\end{abstract}

Electronic supplementary material The online version of this article (doi:10.1007/s00464-012-2549-0) contains supplementary material, which is available to authorized users.

\section{S. Lim}

Sarawak General Hospital, Kuching, Sarawak, Malaysia

e-mail:dr.limms@gmail.com

G. Melich

Department of General Surgery, McGill University, Montreal,

QC, Canada

B. S. Min

Severance Robot and MIS Center, Seoul, Korea

B. S. Min $(\bowtie)$

Department of Surgery, Yonsei University College of Medicine, 250 Seongsanno, Seodaemungu, Seoul 120-752, Korea

e-mail: bsmin@yuhs.ac open surgery and one case was converted to multiport surgery. The mean estimated blood loss was $24.5 \mathrm{ml}$ (range $=5-230$ ), the mean operating time was $167.5 \mathrm{~min}$ (range $=112-251)$, the median skin incision length was $4.7 \mathrm{~cm}$ (range $=4.2-8.0$ ), the mean proximal and distal resection margins were $12.9 \mathrm{~cm}$ (range $=7.5-25.1$ ) and $12.3 \mathrm{~cm}$ (range $=4.5-19.2$ ), respectively, and the mean lymph node harvest was 16.8 (range $=0-42$ ). The immediate postoperative pain score was 2.8 (range $=1-5$ ) and on postoperative day 1 it was 1.4 (range $=1-3$ ). The mean length of hospital stay was 6 days (range $=5-9$ ).

Conclusion Robotic single-incision anterior resection is a safe and viable option for selected patients. Merging the principles of reduced parietal trauma and better cosmesis with the ergonomic advantages of the robotic system is a novel evolution of single-incision laparoscopic surgery.

Keywords Colorectal cancer · Robotic single-incision surgery $\cdot$ Laparoscopic anterior resection $\cdot$ Single-incision anterior resection $\cdot$ Single-incision colectomy

Disclosures Drs. Meng-Shi Lim, George Melich, and Byung Soh Min have no conflicts of interest or financial ties to disclose. 